\title{
Combined high-fat-resveratrol diet and RIP140 knockout mice reveal a novel relationship between elevated bone mitochondrial content and compromised bone microarchitecture, bone mineral mass, and bone strength in the tibia.
}

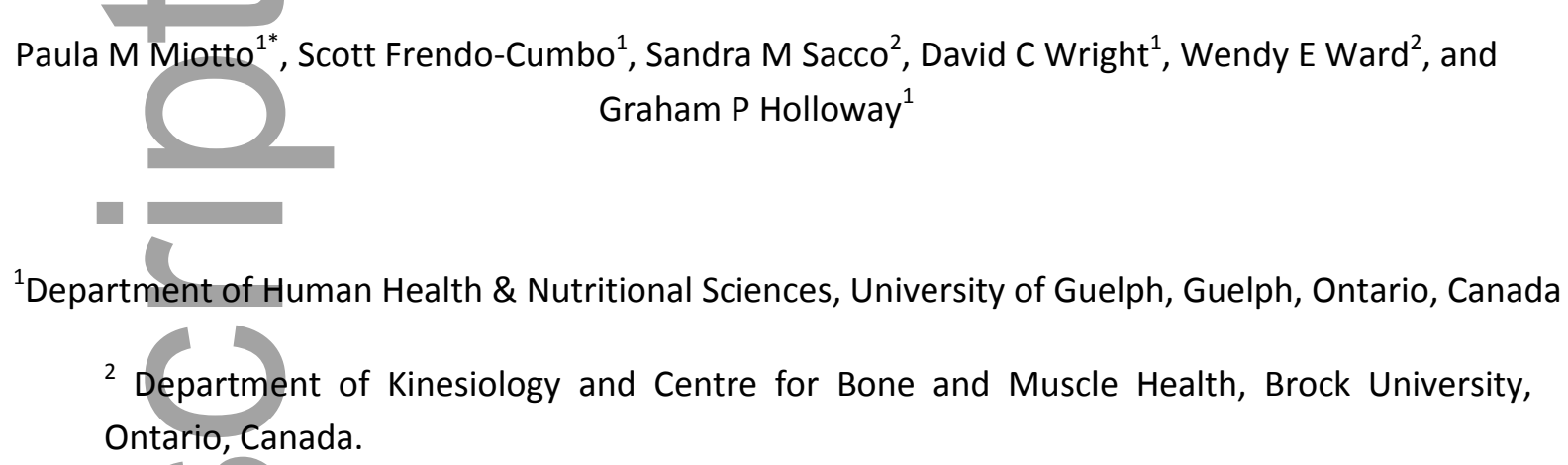

Keywords: bone health, bone turnover, mitochondria, resveratrol, type 2 diabetes.

*Denotes corresponding author:

Paula Miotto

Human Health \& Nutritional Sciences

University of Guelph,

Guelph, Canada

Ph: 519-824-4120 x 56654 Fax: 519-763-5902

Email: pmiotto@uoguelph.ca

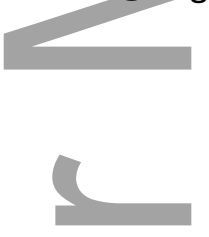

Abbreviations: CON, control; BMC, bone mineral content; BMD, bone mineral density; CTX, carboxyterminal collagen crosslinks; Conn. D, connectivity density; Ct.Ar, cortical bone area; $\mathbf{C t}$. Th, cortical bone thickness; HF, high fat; KO, knockout; OPG, osteoprotegerin; RANKL, receptor activator of nuclear factor kappa-B ligand; RSV, resveratrol; SMI, structural model index; Tb.N, trabecular number; Tb. Sp, trabecular spacing; Tt.Ar, total cross-sectional area; WAT, white adipose tissue.

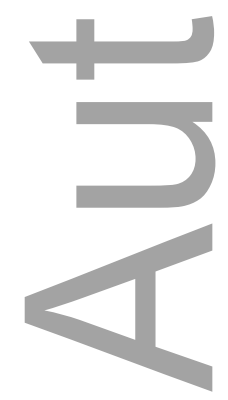

This is the author manuscript accepted for publication and has undergone full peer review but has not been through the copyediting, typesetting, pagination and proofreading process, which may lead to differences between this version and the Version of Record. Please cite this article as doi: 10.1002/mnfr.201500870.

This article is protected by copyright. All rights reserved. 


\section{ABSTRACT}

Scope: While resveratrol (RSV) is associated with the prevention of high-fat (HF) diet-induced insulinresistance, the effects on bone health combined with a HF-diet is unknown. Therefore, we determined the effect of RSV on bone microarchitecture in the presence of a HF-diet, while also elucidating molecular adaptations within bone that could contribute to bone health status. Methods and Results: Male C57BL6 mice were provided control (10\% fat) or HF-diet (60\% fat) in the presence or absence of RSV for 12-weeks. While RSV prevented HF diet-induced glucose-intolerance, HF-RSV compromised tibial microarchitecture, mineral mass, and strength. The compromised outcomes following HF-RSV corresponded with higher markers of osteoclast-activation and bone-resorption (decreased OPG/RANKL ratio; increased cathepsin K), as well as higher markers of tibial mitochondrial content. A molecular model of elevated mitochondrial content (RIP140 knock out (KO) mice) was utilized to determine proof-of-principle that increasing mitochondrial content coincides with decrements in bone health. RIP140 KO mice displayed higher markers of mitochondrial content, and similar to HF-RSV, had compromised bone microarchitecture, lower BMD/strength, and higher markers of osteoclast-activation/bone-resorption. Conclusion: These data show that in the presence of a HF-diet, RSV negatively alters bone health, a process associated with increased mitochondrial content and markers of bone resorption.
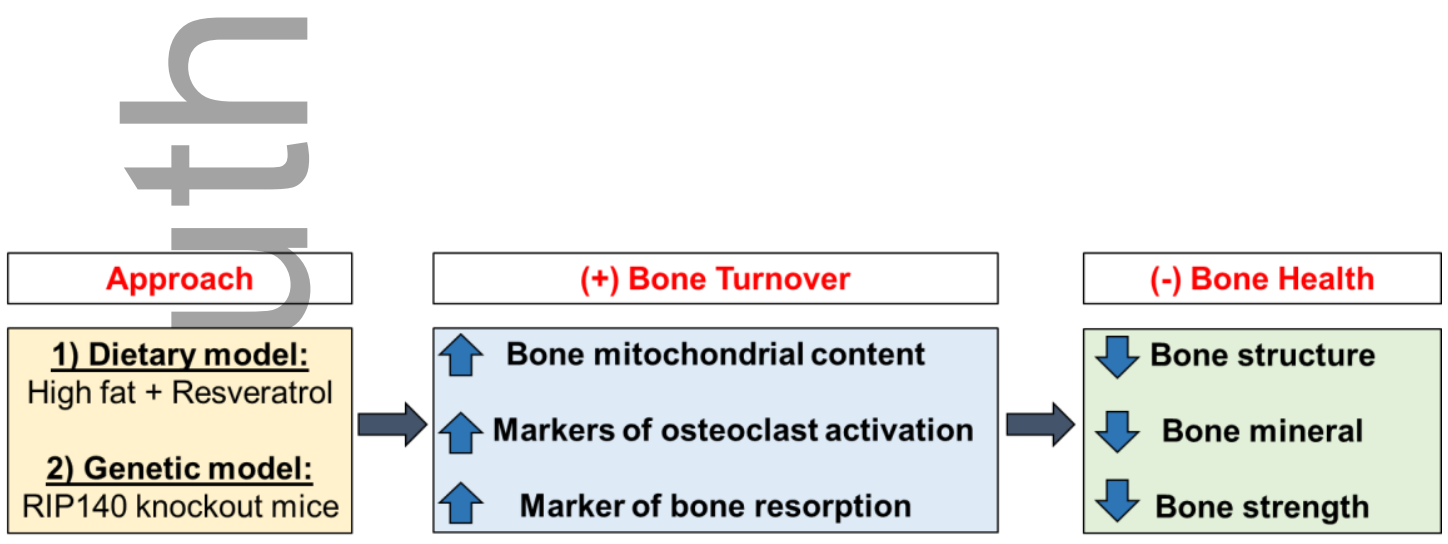

We evaluated the effects of a combined high fat-resveratrol (HF-RSV) diet on bone mitochondrial content and bone health. Our results demonstrated that HF-RSV resulted in increased bone mitochondrial content, which was associated with greater markers of bone

This article is protected by copyright. All rights reserved. 
resorption and weaker bone. We further established a relationship between elevated mitochondrial content and impaired bone health by using RIP140 knockout mice - a model that exhibits increased mitochondrial content. Similar to HF-RSV mice, RIP140 KO mice had increased bone resorption and impaired bone health - establishing a negative association between excess bone mitochondrial content and bone health.
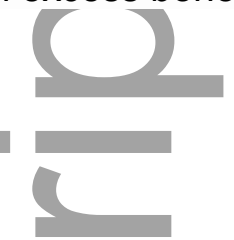

\section{INTRODUCTION}

The prevalence of Type 2 diabetes is increasing at an alarming rate and is a worldwide health concern. The economic burden of this disease is exacerbated by the associated co-morbidities, including osteoporosis [1], which can lead to an increased risk of fragility fracture [2], hospitalization [3], and compromised quality of life [4]. While the cause of Type 2 diabetes remains debatable, a known contributor is the chronic consumption of a high fat (HF) diet, which has been shown to induce weight gain, insulin resistance [5], and is often harmful to bone quantity (bone mineral content or density; BMC, BMD) [6-9] and bone quality (bone microarchitecture; strength) [6, 10-12].

Unfortunately, several current treatments aimed at improving insulin sensitivity have been shown to negatively affect bone quantity and bone quality in rodents $[13,14]$ and humans $[15-17]$. For instance, rosiglitazone can suppress osteoblast differentiation [18] and directly increase osteoclastogenesis $[13,18,19]$, leading to compromised trabecular microarchitecture, BMD and bone strength [18]. Moreover, resveratrol (RSV), a polyphenol associated with several health benefits, including the prevention of HF diet-induced glucose intolerance [5], has been shown to decrease bone mineral mass $[20,21]$, and compromise bone microarchitecture $[20,22]$ and bone strength [21] in aged and physically inactive male rodents. This occurs in contrast to previous reports in female rodents, in which RSV protected against bone loss due to ovariectomy [23-25]. It is currently unknown if these interventions induce molecular alterations within bone that could explain, at least in part, why they negatively impact bone health in male rodents. However, there

This article is protected by copyright. All rights reserved. 
may be common responses in bone that are already established in other tissues with these therapies, thus, providing insight into which interventions are likely to prevent insulin resistance at the expense of bone health.

A conserved response following rosiglitazone and RSV interventions, and occasionally following a HF diet [26], is an increase in mitochondrial content in diverse tissues. For instance, rosiglitazone is a PPAR agonist and increases mitochondrial content in white adipose tissue (WAT) $[25,27]$ and brain $[28]$, while RSV activates the AMPK/SIRT1 axis to influence PGC-1 $\alpha$ mediated gene transcription, and the induction of mitochondrial biogenesis within several tissues, including the heart [23], skeletal muscle [25], WAT [24], and brown adipose tissue [5]. The influence of diet on mitochondrial content within bone remains to be delineated. However, in cell culture, mitochondria are functionally important for osteoblast and osteoclast viability, as well as their capacity to facilitate bone deposition [29] and resorption [30], respectively. Of particular interest, increased mitochondria within osteoclasts is linked with osteoclast differentiation and bone resorption, a highly energetic process [30]. Therefore, an increase in mitochondrial content within bone may coincide with compromised bone health.

The purpose of the current investigation was to determine whether the consumption of RSV increased markers of mitochondrial content (i.e., protein complexes I-V and thus improved oxidative capacity), and perpetuated bone loss in a model of diet-induced glucose intolerance. It was hypothesized that mice consuming a HF diet would have decrements in bone health, responses exacerbated by the combined consumption with RSV. Since RSV and HF consumption can increase mitochondrial content in other peripheral tissues (e.g., adipose, muscle), it was anticipated that compromised bone health would be associated with increased mitochondrial content.

This article is protected by copyright. All rights reserved. 


\section{MATERIALS AND METHODS}

\section{Animals and experimental design}

All experimental protocols complied with the Canadian Council on Animal Care guidelines and were approved by the Animal Care Committee at the University of Guelph. Male C57BL6 mice (8 weeks of age; Charles River) were individually housed with a 12:12 hour light-dark cycle. Mice were randomized ( $n=8-10 /$ group) to either a control (CON; $10 \%$ fat ( $6 \%$ soybean oil; $4 \%$ lard), $70 \%$ carbohydrate (sucrose), $20 \%$ protein (casein); D12450J, Research Diets), high fat (HF; 60\% fat (6\% soybean oil; $54 \%$ lard), $20 \%$ carbohydrate (sucrose), 20\% protein (casein); D12492, Research Diets), or HF-resveratrol (HF-RSV; $100 \mathrm{mg} / \mathrm{kg} /$ day) diet for 12 weeks. RSV was obtained from Cayman Chemicals (100\% trans-resveratrol; CAT\# 70675). Since previous work has shown negligible effects of RSV on basic characteristics/glucose tolerance [5] or bone health [31] in control mice, and the focus ind

of this study was to examine the off-target effects of RSV in the context of diet-induced glucose intolerance, a group receiving control diet combined with RSV was not included.

Provided we are the first to examine mitochondrial content in ex vivo bone samples, it is necessary to utilize an appropriate positive control to strengthen a possible relationship between mitochondrial content and bone health. Thus, to establish whether bone mitochondrial content parallels changes in bone quantity (BMD; $B M C)$ and quality (microarchitecture; strength), we investigated a model known to have high levels of mitochondrial proteins in other tissues. Specifically, we examined bones from RIP140 knockout (KO) mice (male mice; $n=5$ wild-type; $n=5$ KO). RIP140 is a suppressor of PGC-1 $\alpha$ [32]- an important regulator of mitochondrial biogenesis [33]. RIP140 KO mice have higher mitochondrial content in skeletal muscle [33] and adipose [34], similar to the known responses of HF or RSV in the same tissues. Moreover, RIP140 KO mice are resistant to HF diet-induced glucose intolerance [35], similar to combined HF-RSV consumption. Thus, we aimed

This article is protected by copyright. All rights reserved. 
to determine whether bone from RIP140 KO mice also expressed higher mitochondrial content - and whether this corresponded with compromised bone health. Therefore, strengthening a possible relationship between diet-mediated changes in mitochondrial content and bone morphology. Breeding pairs for the RIP140 mouse line were generously provided by Dr. Malcolm Parker.

\section{Glucose tolerance tests, body weight, and anthropometric analysis of the tibia}

Following 12 weeks of feeding, C57BL6 mice (n=6/group) fed the CON, HF and HF-RSV diets were contained in their normal environment and injected intraperitoneally with 2 g glucose $/ \mathrm{kg}$ body weight. Tail blood glucose was monitored using a glucometer at $0,15,30,45,60,90$, and 120 minutes post-injection. These measurements were repeated for RIP140 WT and KO mice. Terminal body weights, food intake, and tibial weights were determined using a balanced scale. Tibiae were measured for longitudinal length, width, and depth using electronic calipers.

\section{Bone selection}

To be consistent with previous literature studying RSV on bone health in males [22], we have chosen to study the tibia as opposed to femur or lumbar vertebrae. Moreover, optimization of protein extraction in our lab has determined to be most effective in the tibia, in terms of successful bone marrow removal and protein yield, and therefore this bone was most appropriate for our analyses.

\section{Bone pulverization and homogenization}

The right tibia was excised from C57BL6 and RIP140 mice and cleared of soft tissue. To eliminate possible interference of mitochondrial content by non-bone related cells (e.g., immune cells), bone marrow was extracted by centrifugation at $16,100 \mathrm{rcf}$ for 20 seconds, a method optimized to be more effective at removing contaminants than traditional saline flushing [36]. Bones were inspected thoroughly following centrifugation, and cleared of any residual marrow. Tibiae were weighed and pulverized under liquid nitrogen using a mortar and pestle. Dried tissue was then homogenized in 15X volume of lysis buffer ( $1 \%$ triton X-100, 50mM Tris-HCL, 1mM EDTA, 1mM EGTA, 50mM NaF,

This article is protected by copyright. All rights reserved. 
$10 \mathrm{mM}$ sodium $\beta$-glycerol phosphate, $5 \mathrm{mM}$ sodium pyrophosphate, $2 \mathrm{mM}$ DTT) with PMSF, sodium orthonavadate, and protease inhibitors (aprotinin, leupeptin, pepstatin A). Supernatant protein concentrations were determined using a Bradford protein assay (Bio-rad). All samples were aliquoted and stored at $-80 \circ \mathrm{C}$.

\section{Trabecular and cortical microarchitecture of tibiae by microcomputed tomography}

Bone morphology and microarchitecture of tibiae was measured using high resolution microcomputed tomography (micro-CT; SkyScan 1176, Bruker-mCT, Kontich, Belgium). The proximal tibia of the left limb was scanned ex vivo at a $9 \mu \mathrm{m}$ resolution and reconstructed using NRecon software (version 1.6.9.10, Bruker-mCT, Kontich, Belgium). Images were reoriented using Dataviewer software (version 1.5.1.3, Bruker-mCT, Kontich, Belgium), and regions of interest were selected using CTA software (version 1.14.4.1+, Bruker-mCT, Kontich, Belgium). Cortical bone measurements included cortical bone area (Ct.Ar), total cross-sectional area (Tt.Ar), cortical area fraction (Ct.Ar/Tt.Ar), and cortical thickness (Ct.Th), and were analysed in 81 successive slices $(0.712 \mathrm{~mm})$ with a separation of 850 slices $(7.476 \mathrm{~mm})$ from the proximal growth plate and spanning into the diaphysis of the tibia. Trabecular structural measurements included bone volume fraction (a ratio of bone volume, BV to total volume, TV), trabecular number (Tb.N), trabecular separation (Tb.Sp), trabecular thickness (Tb.Th), structure model index (SMI), and connectivity density (Conn.D). Trabecular analyses were conducted in 51 successive slices $(0.449 \mathrm{~mm})$, with a separation of 80 slices $(0.704 \mathrm{~mm})$ from the growth plate. Lower and upper grey thresholds were 112 and 255 for cortical bone analyses, and 56 and 255 for trabecular analyses, respectively.

\section{Bone mineral content and density of whole tibia by dual energy X-ray absorptiometry (DXA)}

$\mathrm{BMC}(\mathrm{mg})$ and $\mathrm{BMD}\left(\mathrm{mg} / \mathrm{mm}^{2}\right)$ of whole left tibia were measured using DXA (pSabre, Orthometrix) and specialized software (host software version 3.9.4; scanner software version 1.2.0) [37-39]. Tibiae were scanned in air at a speed of $2 \mathrm{~mm} / \mathrm{s}$ and resolution of $0.1 \times 0.1 \mathrm{~mm}$.

\section{Peak load at the tibial midpoint by a materials testing system}

This article is protected by copyright. All rights reserved. 
Peak load $(\mathrm{N})$, the maximal amount of force a bone can withstand before fracturing, was measured using 3-point bending at the left tibial midpoint with a crosshead speed of $2 \mathrm{~mm} / \mathrm{s}$ (Model 4442 , Instron, Norwood, MA) and analysed using specialized software (Series IX Automated Materials Testing System version 8.1 5.00).

\section{Western blotting}

Tibia homogenates were diluted $(0.2 \mu \mathrm{g} / \mu \mathrm{l})$ and equal amounts of protein $(5 \mu \mathrm{g}$ protein) were loaded for the Western blot detection of $\alpha$-tubulin (1:5000; Abcam, Cambridge, MA, USA), osteoprotegerin (OPG, 1:1000; Abcam), receptor activator of nuclear factor kappa-B ligand (RANKL, 1:1000; Abcam), cathepsin K (1:1000; Abcam), and OXPHOS (1:500; complexes I-V of the mitochondrial electron transport chain; MitoSciences, Eugene, OR, USA). Sample preparation for OXPHOS did not include boiling. Proteins were separated using SDS-PAGE, gels were cut, and all samples for each target of interest were transferred to the same polyvinylidine difluoride membrane. Membranes were blocked and incubated with appropriate primary and secondary antibodies. Each membrane was treated with chemiluminescence solution (Western Lightning, plus ECL; PerkinElmer, Inc, Waltham, MA) and detected using enhanced chemiluminesence (ChemiGenius2 Bioimaging system, SynGene, Cambridge, UK).

\section{Statistical Analyses}

Data from CON, HF, and HF-RSV mice were analyzed using one-way ANOVA followed by StudentNewman Keuls post-hoc analysis (SigmaPlot, version 12.0, Systat Software Inc, San Jose, CA). Food intakes from HF mice (HF; HF-RSV) and data from RIP140 wild-type and KO mice were analyzed using independent samples t-tests (one-tailed, mitochondrial markers; two-tailed, bone outcomes). Associations between total mitochondrial content (sum of complex I-V per mouse) and main morphological outcomes (i.e., BMC, BV / TV, peak load, cathepsin K) were determined using Pearson $r$ correlation analyses. Statistical significance was determined at $\mathrm{P} \leq 0.05$.

\section{RESULTS}

This article is protected by copyright. All rights reserved. 


\section{Mice fed a CON, HF, or HF-RSV diet}

\section{Glucose tolerance tests, body weight, and bone anthropometric measurements}

We first aimed to verify the expected responses to the dietary interventions. As expected, the glucose area under the curve $(\mathrm{AUC})$ was $\sim 35 \%$ higher $(\mathrm{P}<0.05)$ after consuming HF relative to CON, while HF-RSV mice displayed a normal response (Figure 1). There were no differences in food intake between mice fed HF and HF-RSV (Table 1). In addition, the mice fed a HF diet weighed (g) more than CON and HF-RSV mice (Table 1). However, there were no differences in tibial length, width, depth, or weight (Table 1). Given the appropriate responses to HF diet and HF-RSV we next aimed to determine the impact of these diets on bone health, and the association with mitochondrial content.

\section{Cortical and trabecular microarchitecture, bone mineral mass, and bone strength in the tibia}

Consumption of a HF diet did not alter the cortical (Table 1) or trabecular (Figure 2. A-H) tibial microarchitecture. Moreover, bone mineral and peak load of the tibial midpoint were not altered following a HF diet (Figure 3. A-C). In contrast, HF-RSV compromised several indices of bone health. Specifically, while cortical indices were not altered (Table 1), HF-RSV consumption compromised trabecular microarchitecture, as tibiae from these mice displayed higher trabecular separation and lower Tb. BV/TV (\%), Tb. N, and Conn.D than CON and HF fed mice (Figure 2. DH). In addition, mice fed a HF-RSV diet also had lower tibial BMC (Figure 3. A) and BMD (Figure 3. B), as well as $\sim 25 \%$ less force required to fracture the tibial midpoint in these mice (Figure $3 . \mathrm{C}$ ). Altogether, the consumption of RSV during a HF challenge compromised several indices of bone health.

\section{Protein markers of osteoclast activation and bone resorption in the tibia}

Provided there were no differences in bone anthropometric measures between groups, it was not suspected that bone growth was impaired. Therefore, we next determined whether the morphological findings are related to bone turnover, particularly bone resorption. The consumption of a HF diet did not alter the ratio of OPG to RANKL or cathepsin K protein content (Figure 3. D, E), suggesting unaltered osteoclast activation, and supporting the observed morphological responses. In

This article is protected by copyright. All rights reserved. 
contrast, the consumption of RSV in the presence of a HF-diet resulted in a lower ratio of OPG to RANKL (Figure 3. D) and higher cathepsin $\mathrm{K}$ content (Figure 3. E). Together, these data suggest enhanced osteoclast activation and bone resorption following HF-RSV, responses which may contribute, at least partly, to the compromised trabecular microarchitecture, lower BMC and BMD, as well as bone strength at the tibial midpoint.

\section{Protein markers of mitochondrial content in the tibia}

Previous work in tissues such as skeletal muscle [5], adipose [24], and heart [23] have shown elevated mitochondrial content with RSV - a response that has not been studied in bone. Since our data suggests an increase in bone turnover, we next aimed to determine whether bone oxidative capacity could be influenced by HF-RSV, perhaps as a way to support bone turnover. Similar to markers of bone microarchitecture, mineral mass, and osteoclast activation, the consumption of a HF diet did not alter markers of mitochondrial content (Figure 4. A-F). In contrast, HF-RSV consistently increased the protein content of electron transport chain subunits $~ 30 \%$ (Figure 4 . A-F). Altogether, these data suggest HF-RSV induced mitochondrial biogenesis in association with markers of osteoclast activation and compromised bone health.

\section{A molecular model of elevated mitochondrial content: RIP140 knockout mice}

\section{Protein markers of mitochondrial content in the tibia}

Overall, our data indicates that consumption of the HF-RSV diet increased mitochondrial content in the tibia, a response associated with compromised bone health. Therefore, we next aimed to directly determine if elevations in bone mitochondrial content mitigate glucose intolerance at the expense of bone health using a model of elevated mitochondrial content.

We are the first to characterize the effect of altering RIP140 abundance on mitochondrial content within bone. Ablation of the RIP140 gene increased several markers of mitochondrial content in the tibia by $\sim 50 \%$. Specifically, subunits of complexes I-V were higher in RIP140 KO mice compared to wild-type controls (Figure 5. A-F). As expected [40], RIP140 KO mice had higher glucose tolerance (i.e., better ability to dispose of circulating glucose into peripheral tissues) than wild-type (data not

This article is protected by copyright. All rights reserved. 
shown). However, there were no differences in tibial length, width, depth, or weight (Table 2). Therefore, we next determined whether RIP140 KO mice displayed impaired bone microarchitecture, mineral mass, and bone strength.

\section{Cortical and trabecular microarchitecture, bone mineral mass, and bone strength in the tibia}

RIP140 wild-type and KO mice had similar cortical indices of bone microarchitecture. This included Tt.Ar, Ct.Ar, Ct.Ar/Tt.Ar (\%), and Ct. Th (Table 2). However, RIP140 KO mice had significantly lower Tb. BV/TV(\%), Tb.N, and Conn. D, and higher Tb. Sp (Figure 6. A-G) and SMI (Table 2), without changes in Tb.Th (Figure 6. E). In addition, RIP140 KO mice had lower BMD (Figure 7. A) and peak load at the tibial midpoint (Figure 7. C). Collectively, these results indicate that elevated mitochondrial content was consistently and inversely associated with compromised bone microarchitecture, lower bone mineral mass, and lower bone strength at the tibial midpoint.

\section{Protein markers of osteoclast activation and bone resorption in the tibia}

In RIP140 KO mice, the increase in mitochondrial content coincided with a $25 \%$ lower ratio of OPG to RANKL, and $\sim 40 \%$ higher cathepsin $\mathrm{K}$ protein content (Figure $7 . \mathrm{D}, \mathrm{E}$ ), similar to the observed responses in HF-RSV mice. These data indicate that increased mitochondrial content mirrored higher markers of osteoclast activation and bone resorption in the tibia, which suggests that a possible common denominator with the effects of RSV in bone, as reported in other tissues, may be an increase in mitochondrial content. Together, both models display elevated bone mitochondrial content and compromised bone microarchitecture, mineral mass, and bone strength.

Elevated mitochondrial content is associated with decrements in bone mineral, microarchitecture, and strength

We wanted to further evaluate the relationship between mitochondrial content and decrements in bone health, and thus performed Pearson $r$ correlational analyses. There were strong negative associations between mitochondrial content and BMC, BV/TV, and peak load (Figure 8. A-C). 
Furthermore, there was a strong positive correlation between mitochondrial content and cathepsin K (Figure 8. D).

DISCUSSION

Combined, the current data shows that the consumption of HF-RSV is negatively associated with bone health. In addition, we are the first to provide evidence that HF-RSV mediated decrements in bone health coincide with increased mitochondrial content and markers of osteoclast activation and bone resorption within the tibia. Moreover, we demonstrate that higher bone mitochondrial content, in the absence of dietary intervention, also corresponds with compromised bone trabecular microarchitecture, whole bone $\mathrm{BMC}$ and $\mathrm{BMD}$, and bone strength at the tibial midpoint. Collectively, these data provide molecular insight into how a nutraceutical, such as RSV, that is beneficial for one aspect of health when combined with a HF diet can negatively affect bone health. These data also suggests the oxidative capacity of bone is an important variable to consider in preventive approaches aimed at mitigating insulin resistance and glucose intolerance.

Compromised bone microarchitecture, bone mineral mass, and bone strength coincides with increased markers of mitochondrial content in the tibia

The current study demonstrates that diet-induced glucose intolerance does not by itself compromise bone health. Our findings that HF consumption did not alter BMD, bone microarchitecture, or bone strength compared to controls is supported by some [37, 41] but not all [6-12] previous studies. A possible explanation could be the mixed composition of diet used (e.g., lard and soybean oil), in which the presence of soybean oil negated any effect on bone mitochondrial content or bone health. Interestingly, HF-RSV resulted in compromised both health despite similar body weight as CON and dietary intake as HF. Therefore, indicating that the effects were mediated through the combined HF-RSV intervention and not nutrient deficiency. Specifically, mice fed HF-RSV $(100 \mathrm{mg} / \mathrm{kg} /$ day) positively mitigated $\mathrm{HF}$ diet induced glucose intolerance, however, trabecular bone 
microarchitecture was compromised and accompanied by lower BMC, BMD, and lower force required to fracture the tibial midpoint than mice fed CON or HF diet. Although cortical structure in both HF-RSV and RIP140 mice are not different from their respective controls, they both exhibit lower bone strength (measured as peak load) at the tibial midpoint. While a limitation is that we were unable to obtain $\mathrm{BMC}$ and $\mathrm{BMD}$ by micro computed tomography at the cortical sites where bone structure and strength were measured, it is possible that these measures were lower and could explain decrements in bone strength despite similar outcomes for cortical structure. In this regard, estimates of mineral measurements using micro-CT can exhibit some degree of error [42], while DXA cannot differentiate mineral content within separate compartments [43]. Further, we cannot exclude a possible interactive effect of HF and RSV on bone health, due to the absence of a CON-RSV group. However, our data is supported by previous reports in both aging [22] and physically inactive male rats $[20,21]$ that also demonstrated deleterious effects of RSV $(12.5-20 \mathrm{mg} / \mathrm{kg} / \mathrm{day})$ on tibial bone microarchitecture, mineral mass, and strength - in the absence of a HF challenge. Although not all models respond similarly to RSV, as female ovariectomized (OVX) mice display protective effects of RSV ( $\sim 80 \mathrm{mg} / \mathrm{kg} /$ day) on bone health [44-46], our data demonstrates a detrimental effect of RSV in male mice challenged with a HF diet. It is currently unknown why such divergent responses to RSV occur in different models, but it could be related to factors including age, sex hormones, or internal (ovariectomy) versus environmental (i.e., diet, physical inactivity) challenges. Interestingly, previous work using the OVX model has shown reduced mitochondrial content and function in brain [47] and skeletal muscle [48], respectively. Therefore, although speculative, it is possible that OVX rodents have lower bone mitochondrial content and lower bone 'turnover', contributing to weaker bones. In contrast, perhaps RSV could assist in attenuating bone loss due to ovariectomy by normalizing mitochondrial content (i.e., promoting higher mitochondria, as our data demonstrates). Therefore, a possible 'inverted U relationship' may be evident between bone mitochondrial content and bone health across different rodent models exhibiting low, normal, and high levels of mitochondria. Clearly future work should evaluate bone mitochondrial alterations upon OVX and combined OVX-

This article is protected by copyright. All rights reserved. 
RSV, as the current study provides novel insight that mitochondria could be a consideration for bone health.

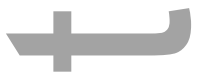

It is important to highlight that neither HF-RSV nor RIP140 KO mice had alterations in bone size (i.e., length, width, depth), weight, or total volume analysed by micro-CT. Therefore, the absolute measurements regarding bone mineral mass, microarchitecture, and bone strength can be directly compared - as these findings indicate skeletal growth was not impaired. Thus, the lower amount of bone mineral and compromised bone microarchitecture and strength are reflective of changes in bone turnover, each of which is strongly correlated to greater oxidative capacity (i.e., higher mitochondrial protein contents) to fuel bone turnover, rather than simply being smaller bones. Interestingly, lower bone mineral, compromised bone microarchitecture, and lower midpoint bone strength with RSV coincided with $\sim 30 \%$ higher protein content of the electron transport chain complexes. In support of a potential link between elevated mitochondrial content and decrements in bone health, RIP140 KO mice also exhibited higher mitochondrial protein contents of complexes I-V, which corresponded with lower tibial BMD, strength, BV/TV (\%), trabecular number, and connectivity density; along with higher trabecular separation and SMI - findings of which mirrored those for mice fed HF-RSV. Although complex II in HF-RSV mice is not statistically significant, this follows the same pattern of change as complexes I and III-V. Moreover, total mitochondrial content $(\mathrm{Cl}-\mathrm{V})$ is strongly correlated with decrements in bone health and cathepsin $\mathrm{K}$, therefore, the cumulative elevation in mitochondrial markers are physiologically important. Thus, it is apparent that the compromised bone health with combined HF-RSV is associated with elevations in bone mitochondrial content.

HF-RSV and RIP140 KO mice have elevated markers of osteoclast activation and bone resorption in the tibia.

HF-RSV mediated loss of bone mineral, compromised bone microarchitecture, and lower strength could be due to a combination of lower osteoblast bone deposition and/or increased osteoclast

This article is protected by copyright. All rights reserved. 
bone resorption [49]. Although this study does not delineate which cell types are represented by the altered mitochondrial content, previous work has indicated that RSV increased CTX, a serum marker of bone resorption, without significant alterations in alkaline phosphatase (bone formation)[22]. In the current study, we show that increased mitochondrial content (HF-RSV and RIP140 KO) is associated with higher markers of osteoclast activation and bone resorption. Specifically, the ratio of OPG to RANKL was used as a marker of osteoclast activation from inactive precursors [50], while cathepsin K, an important protease highly expressed and secreted by osteoclasts, was also measured. It has been established that cathepsin $\mathrm{K}$ is critical for bone resorption, as ablation from osteoclasts results is osteopetrosis - a condition characterized by excess bone mineralization due to impaired osteoclast activation and severe impairments in bone resorption [51]. Importantly, we measured these proteins directly within the bone, as opposed to the blood, as OPG can also be secreted by liver, kidney, and heart [52]. Therefore, the observed lower ratio of OPG to RANKL following HF-RSV and RIP140 ablation suggest greater osteoclast activation within the bone, a response accompanied by higher cathepsin $\mathrm{K}$ protein contents in both mouse models. Osteoclasts are large multinucleated cells known to have high mitochondrial contents and energy demands [30], suggesting that HF-RSV mediated elevation in mitochondrial content could reflect increased osteoclast content. In support of this, the insulin sensitizer rosiglitazone, which is known to increase mitochondrial content in diverse tissues $[25,27]$, can primarily increase osteoclastogenesis within bone [13]. Although a concerted response to HF-RSV in osteoblasts and osteocytes could also occur, our data indicates that increased bone mitochondrial content is associated with higher markers of bone resorption, and this could partly explain the decrements in bone health. Although it is unknown whether bone formation markers are altered with HF-RSV feeding, this would not affect our interpretation that HF-RSV led to decrements in bone health that are associated with increased markers of bone resorption. Together, these findings support the supposition that decrements in bone health demonstrated in HF-RSV and RIP140 KO mice correspond with an increased oxidative

This article is protected by copyright. All rights reserved. 
capacity, which may be a molecular adaptation used to match the high energy demands of bone turnover in the tibia.

\section{Concluding Remarks}

The current data indicate that RSV successfully mitigated HF diet induced weight gain and glucose intolerance, however, this occurred at the expense of bone microarchitecture, bone mineral, and bone strength. Although we do not demonstrate that RSV alone alters bone mitochondrial content or bone morphology, this does not detract from the novel relationship established between mitochondrial content and bone health. In this regard, it appears that a possible predictor for the negative effect of interventions such as HF-RSV could involve an increase in mitochondrial content, as RIP140 KO mice mirrored the changes observed following HF-RSV. Several pharmacological interventions (e.g., pioglitazone, metformin) have been shown to affect oxidative phosphorylation in other tissues, and therefore should be evaluated for off target effects on bone health, as should future therapeutic strategies for insulin resistance. This knowledge is particularly important considering the aging population, and the growing number of individuals consuming insulinsensitizing agents whose bone health may be compromised. Such individuals could be at a heightened risk of osteoporosis and associated debilitating fragility fractures. However, caution is warranted with respect to directly translating rodent research to humans as a high level of fat and RSV was provided. Also, since the dietary interventions began during early life, when rapid rates of growth and maturation occur, future study could elucidate if similar effects are observed when such interventions are introduced at later stages of the lifespan. Therefore, while the present data highlights a novel relationship between mitochondrial content and bone health, further investigations in clinical populations are required to fully elucidate the consequences of HF-RSV on overall bone health.

\section{Author Contributions}

This article is protected by copyright. All rights reserved. 
P.M.M, S. FC and S.M.S. conducted experimental work and analyzed data, while P.M.M, D.C.W,

W.E.W. and G.P.H conceptualized the study and designed the experiments. All authors contributed to the interpretation of the results and editing the manuscript. P.M.M. and G.P.H. wrote the manuscript. G.P.H is guarantor of this work.

\section{Acknowledgements}

P.M.M and S.FC are recipients of NSERC graduate scholarships. D.C.W holds a Canada Research Chair in Lipids, Metabolism, and Health and W.E.W holds a Canada Research Chair in Bone and Muscle Development. This research was funded by the Natural Sciences and Engineering Research Council of Canada (NSERC) (G.P.H, D.C.W) and the Ontario Ministry of Agriculture, Food and Rural Affairs (OMAFRA) to D.C.W with support for purchase of the microcomputed tomography system from the Canada Foundation for Innovation (W.E.W).

\section{Conflict of Interest}

The authors declare no conflict of interest.

\section{REFERENCES}

[1] Rakel, A., Sheehy, O., Rahme, E., LeLorier, J., Osteoporosis among patients with type 1 and type 2 diabetes. Diabetes \& metabolism 2008, 34, 193-205.

[2] Nicodemus, K. K., Folsom, A. R., Type 1 and type 2 diabetes and incident hip fractures in postmenopausal women. Diabetes care 2001, 24, 1192-1197.

[3] Tarride, J. E., Hopkins, R. B., Leslie, W. D., Morin, S., et al., The burden of illness of osteoporosis in Canada. Osteoporosis international : a journal established as result of cooperation between the European Foundation for Osteoporosis and the National Osteoporosis Foundation of the USA 2012, 23, 2591-2600.

[4] Borgstrom, F., Lekander, I., Ivergard, M., Strom, O., et al., The International Costs and Utilities Related to Osteoporotic Fractures Study (ICUROS)--quality of life during the first 4 months after fracture. Osteoporosis international : a journal established as result of cooperation between the European Foundation for Osteoporosis and the National Osteoporosis Foundation of the USA 2013, 24, 811-823.

[5] Lagouge, M., Argmann, C., Gerhart-Hines, Z., Meziane, H., et al., Resveratrol improves mitochondrial function and protects against metabolic disease by activating SIRT1 and PGC-1alpha. Cell 2006, 127, 1109-1122.

[6] Bielohuby, M., Matsuura, M., Herbach, N., Kienzle, E., et al., Short-term exposure to lowcarbohydrate, high-fat diets induces low bone mineral density and reduces bone formation in rats. Journal of bone and mineral research : the official journal of the American Society for Bone and Mineral Research 2010, 25, 275-284.

This article is protected by copyright. All rights reserved. 
[7] Chen, J. R., Lazarenko, O. P., Wu, X., Tong, Y., et al., Obesity reduces bone density associated with activation of PPARgamma and suppression of $\mathrm{Wnt} /$ beta-catenin in rapidly growing male rats. PloS one 2010, 5, e13704.

[8] Parhami, F., Tintut, Y., Beamer, W. G., Gharavi, N., et al., Atherogenic high-fat diet reduces bone mineralization in mice. Journal of bone and mineral research : the official journal of the American Society for Bone and Mineral Research 2001, 16, 182-188.

[9] Xiao, Y., Cui, J., Li, Y. X., Shi, Y. H., et al., Dyslipidemic high-fat diet affects adversely bone metabolism in mice associated with impaired antioxidant capacity. Nutrition 2011, 27, 214-220.

[10] Cao, J.J., Gregoire, B. R., Gao, H., High-fat diet decreases cancellous bone mass but has no effect on cortical bone mass in the tibia in mice. Bone 2009, 44, 1097-1104.

[11] Patsch, J. M, Kiefer, F. W., Varga, P., Pail, P., et al., Increased bone resorption and impaired bone microarchitecture in short-term and extended high-fat diet-induced obesity. Metabolism: clinical and experimental 2011, 60, 243-249.

[12] Wohl, G. R., Loehrke, L., Watkins, B. A., Zernicke, R. F., Effects of high-fat diet on mature bone mineral content, structure, and mechanical properties. Calcified tissue international 1998, 63, 74-79. [13] Lazarenko, O. P., Rzonca, S. O., Hogue, W. R., Swain, F. L., et al., Rosiglitazone induces decreases in bone mass and strength that are reminiscent of aged bone. Endocrinology 2007, 148, 2669-2680.

[14] Rzonca, S. O., Suva, L. J., Gaddy, D., Montague, D. C., Lecka-Czernik, B., Bone is a target for the antidiabetic compound rosiglitazone. Endocrinology 2004, 145, 401-406.

[15] Bilezikian, J. P., Josse, R. G., Eastell, R., Lewiecki, E. M., et al., Rosiglitazone decreases bone mineral density and increases bone turnover in postmenopausal women with type 2 diabetes mellitus. The Journal of clinical endocrinology and metabolism 2013, 98, 1519-1528.

[16] Kahn, S. E., Haffner, S. M., Viberti, G., Herman, W. H., et al., Rosiglitazone decreases C-reactive protein to a greater extent relative to glyburide and metformin over 4 years despite greater weight gain: observations from a Diabetes Outcome Progression Trial (ADOPT). Diabetes care 2010, 33, 177183.

[17] Schwartz, A. V., Sellmeyer, D. E., Vittinghoff, E., Palermo, L., et al., Thiazolidinedione use and bone loss in older diabetic adults. The Journal of clinical endocrinology and metabolism 2006, 91, 3349-3354.

[18] Sardone, L. D., Renlund, R., Willett, T. L., Fantus, I. G., Grynpas, M. D., Effect of rosiglitazone on bone quality in a rat model of insulin resistance and osteoporosis. Diabetes 2011, 60, 3271-3278. [19] Wei, W., Wang, X., Yang, M., Smith, L. C., et al., PGC1beta mediates PPARgamma activation of osteoclastogenesis and rosiglitazone-induced bone loss. Cell metabolism 2010, 11, 503-516. [20] Durbin, S. M., Jackson, J. R., Ryan, M. J., Gigliotti, J. C., et al., Resveratrol supplementation influences bone properties in the tibia of hindlimb-suspended mature Fisher 344 x Brown Norway male rats. Applied physiology, nutrition, and metabolism = Physiologie appliquee, nutrition et metabolisme 2012, 37, 1179-1188.

[21] Wright, S., Jackson, JR, Ryan, MJ, Alway, SE, Tou, JC., Resveratrol Affects Bone Minerals and Strength in Aging Hindlimb Suspended Rats. FASEB journal : official publication of the Federation of American Societies for Experimental Biology 2010, 24.

[22] Lee, A. M., Shandala, T., Nguyen, L., Muhlhausler, B. S., et al., Effects of resveratrol supplementation on bone growth in young rats and microarchitecture and remodeling in ageing rats. Nutrients 2014, 6, 5871-5887.

[23] Csiszar, A., Labinskyy, N., Pinto, J. T., Ballabh, P., et al., Resveratrol induces mitochondrial biogenesis in endothelial cells. American journal of physiology. Heart and circulatory physiology 2009, 297, H13-20.

[24] Beaudoin, M. S., Snook, L. A., Arkell, A. M., Simpson, J. A., et al., Resveratrol supplementation improves white adipose tissue function in a depot-specific manner in Zucker diabetic fatty rats. American journal of physiology. Regulatory, integrative and comparative physiology 2013, 305, R542-551.

This article is protected by copyright. All rights reserved. 
[25] Wilson-Fritch, L., Nicoloro, S., Chouinard, M., Lazar, M. A., et al., Mitochondrial remodeling in adipose tissue associated with obesity and treatment with rosiglitazone. The Journal of clinical investigation 2004, 114, 1281-1289.

[26] Jain, S. S., Paglialunga, S., Vigna, C., Ludzki, A., et al., High-fat diet-induced mitochondrial biogenesis is regulated by mitochondrial-derived reactive oxygen species activation of CaMKII. Diabetes 2014, 63, 1907-1913.

[27] Rong, J. X., Qiu, Y., Hansen, M. K., Zhu, L., et al., Adipose mitochondrial biogenesis is suppressed in $\mathrm{db} / \mathrm{db}$ and high-fat diet-fed mice and improved by rosiglitazone. Diabetes 2007, 56, 1751-1760. [28] Strum, J. C., Shehee, R., Virley, D., Richardson, J., et al., Rosiglitazone induces mitochondrial biogenesis in mouse brain. Journal of Alzheimer's disease : JAD 2007, 11, 45-51.

[29] Komarova, S. V., Ataullakhanov, F. I., Globus, R. K., Bioenergetics and mitochondrial transmembrane potential during differentiation of cultured osteoblasts. American journal of physiology. Cell physiology 2000, 279, C1220-1229.

[30] Ishii, K. A., Fumoto, T., Iwai, K., Takeshita, S., et al., Coordination of PGC-1beta and iron uptake in mitochondrial biogenesis and osteoclast activation. Nature medicine 2009, 15, 259-266.

[31] Habold, C., Momken, I., Ouadi, A., Bekaert, V., Brasse, D., Effect of prior treatment with resveratrol on density and structure of rat long bones under tail-suspension. Journal of bone and mineral metabolism 2011, 29, 15-22.

[32] Hallberg, M., Morganstein, D. L., Kiskinis, E., Shah, K., et al., A functional interaction between RIP140 and PGC-1alpha regulates the expression of the lipid droplet protein CIDEA. Molecular and cellular biology 2008, 28, 6785-6795.

[33] Seth, A., Steel, J. H., Nichol, D., Pocock, V., et al., The transcriptional corepressor RIP140 regulates oxidative metabolism in skeletal muscle. Cell metabolism 2007, 6, 236-245.

[34] Leonardsson, G., Steel, J. H., Christian, M., Pocock, V., et al., Nuclear receptor corepressor RIP140 regulates fat accumulation. Proceedings of the National Academy of Sciences of the United States of America 2004, 101, 8437-8442.

[35] Christian, M., White, R., Parker, M. G., Metabolic regulation by the nuclear receptor corepressor RIP140. Trends in endocrinology and metabolism: TEM 2006, 17, 243-250.

[36] Kelly, N. H., Schimenti, J. C., Patrick Ross, F., van der Meulen, M. C., A method for isolating high quality RNA from mouse cortical and cancellous bone. Bone 2014, 68, 1-5.

[37] Miotto, P. M., Castelli, L. M., Amoye, F., LeBlanc, P. J., et al., Maternal high fat feeding does not have long-lasting effects on body composition and bone health in female and male Wistar rat offspring at young adulthood. Molecules 2013, 18, 15094-15109.

[38] Sacco, S. M., Jiang, J. M., Reza-Lopez, S., Ma, D. W., et al., Flaxseed does not antagonize the effect of ultra-low-dose estrogen therapy on bone mineral density and biomechanical bone strength in ovariectomized rats. Journal of toxicology and environmental health. Part A 2009, 72, 1209-1216. [39] Kaludjerovic, J., Ward, W. E., Neonatal exposure to daidzein, genistein, or the combination modulates bone development in female CD-1 mice. The Journal of nutrition 2009, 139, 467-473. [40] Powelka, A. M., Seth, A., Virbasius, J. V., Kiskinis, E., et al., Suppression of oxidative metabolism and mitochondrial biogenesis by the transcriptional corepressor RIP140 in mouse adipocytes. The Journal of clinical investigation 2006, 116, 125-136.

[41] Lau, B. Y., Fajardo, V. A., McMeekin, L., Sacco, S. M., et al., Influence of high-fat diet from differential dietary sources on bone mineral density, bone strength, and bone fatty acid composition in rats. Applied physiology, nutrition, and metabolism = Physiologie appliquee, nutrition et metabolisme 2010, 35, 598-606.

[42] Bouxsein, M. L., Boyd, S. K., Christiansen, B. A., Guldberg, R. E., et al., Guidelines for assessment of bone microstructure in rodents using micro-computed tomography. Journal of bone and mineral research : the official journal of the American Society for Bone and Mineral Research 2010, 25, 14681486.

[43] Beck, T., Measuring the structural strength of bones with dual-energy X-ray absorptiometry: principles, technical limitations, and future possibilities. Osteoporosis international : a journal

This article is protected by copyright. All rights reserved. 
established as result of cooperation between the European Foundation for Osteoporosis and the National Osteoporosis Foundation of the USA 2003, 14 Suppl 5, S81-88.

[44] Zhao, H., Li, X., Li, N., Liu, T., et al., Long-term resveratrol treatment prevents ovariectomyinduced osteopenia in rats without hyperplastic effects on the uterus. The British journal of nutrition 2014, 111, 836-846.

[45] Lin, Q., Huang, Y. M., Xiao, B. X., Ren, G. F., Effects of resveratrol on bone mineral density in ovarectomized rats. International journal of biomedical science : IJBS 2005, 1, 76-81.

[46] Liu, Z. P., Li, W. X., Yu, B., Huang, J., et al., Effects of trans-resveratrol from Polygonum cuspidatum on bone loss using the ovariectomized rat model. Journal of medicinal food 2005, 8, 1419.

[47] Xu, X. W., Shi, C., He, Z. Q., Ma, C. M., et al., Effects of phytoestrogen on mitochondrial structure and function of hippocampal CA1 region of ovariectomized rats. Cellular and molecular neurobiology 2008, 28, 875-886.

[48] Cavalcanti-de-Albuquerque, J. P., Salvador, I. C., Martins, E. L., Jardim-Messeder, D., et al., Role of estrogen on skeletal muscle mitochondrial function in ovariectomized rats: a time course study in different fiber types. J Appl Physiol (1985) 2014, 116, 779-789.

[49] Feng, X., McDonald, J. M., Disorders of bone remodeling. Annual review of pathology 2011, 6, 121-145.

[50] Boyle, W. J., Simonet, W. S., Lacey, D. L., Osteoclast differentiation and activation. Nature 2003, $423,337-342$.

[51] Saftig, P., Hunziker, E., Wehmeyer, O., Jones, S., et al., Impaired osteoclastic bone resorption leads to osteopetrosis in cathepsin-K-deficient mice. Proceedings of the National Academy of Sciences of the United States of America 1998, 95, 13453-13458.

[52] Boyce, B. F., Xing, L., Functions of RANKL/RANK/OPG in bone modeling and remodeling. Archives of biochemistry and biophysics 2008, 473, 139-146.

\section{Figure Legends}

Figure 1. Glucose tolerance tests between mice fed CON, HF, or HF-RSV diet.

a) Glucose concentrations, b) AUC. Mice fed a HF diet had higher AUC and were glucose intolerant compared to CON mice. HF-RSV mice prevented diet induced glucose intolerance. Data is expressed as mean \pm SEM.

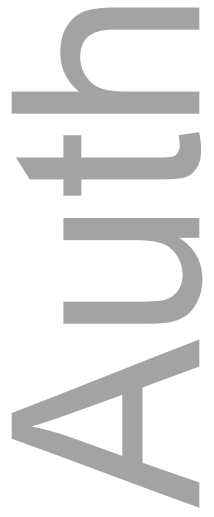

This article is protected by copyright. All rights reserved. 
A)
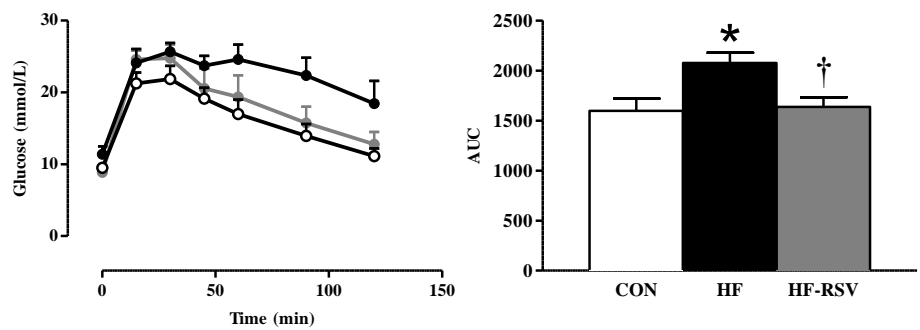

Figure 1

Figure 2. Trabecular bone microarchitecture in the tibia of mice fed a CON, HF, or HF-RSV diet. a-c) representative micro-CT images, d) Tb. BV/TV(\%), e) Tb. N, f) Tb.Th, g) Tb.Sp, h) conn.D. Mice consuming a HF-RSV diet had higher trabecular separation than mice fed a CON diet. CON, control; HF, high fat; HF-RSV, high-fat-resveratrol; Tb. BV/TV, bone volume/total volume; Tb. N, trabecular number; Tb.Th, trabecular thickness; Tb.Sp, trabecular separation; conn.D, connectivity density. *, significantly different from CON. Data is expressed as mean \pm SEM. $n=7-10 /$ group.

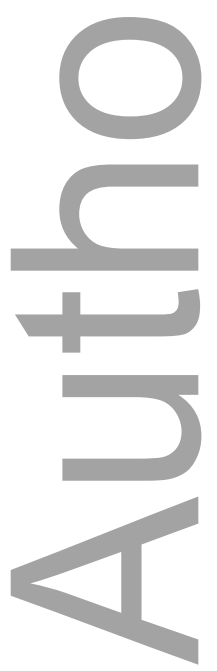

This article is protected by copyright. All rights reserved. 

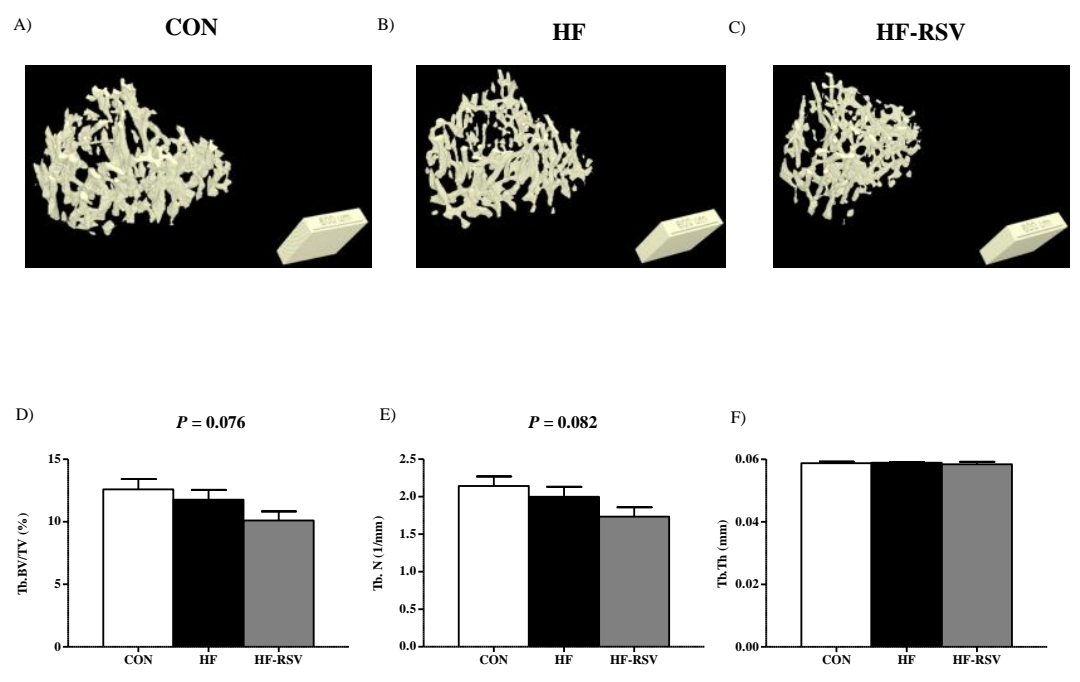

Figure 2

Figure 3. Bone mineral mass, bone strength, and markers of osteoclast activation and bone resorption in the tibia of mice fed a CON, HF, or HF-RSV diet. a) BMC, b) BMD, c) peak load at the tibial midpoint, d) OPG/RANKL (osteoclast activation) and e) cathepsin K (bone resorption). Mice receiving a HF-RSV diet had lower BMD, BMC, and peak load at the tibial midpoint. Mice receiving a HF-RSV diet also had a lower OPG/RANKL ratio and higher cathepsin $\mathrm{K}$ than mice fed CON or HF diet. CON, control; HF, high fat; HF-RSV, high-fat-resveratrol; BMC, bone mineral content; $\mathrm{BMD}$, bone mineral density. ${ }^{*}$, significantly different from CON. ${ }^{+}$, significantly different from HF. $\mathrm{n}=7-$ 10/group.

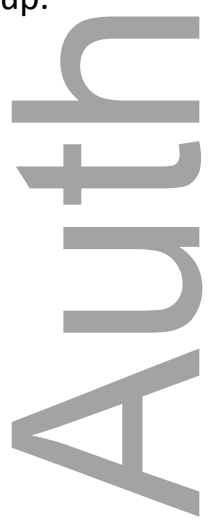



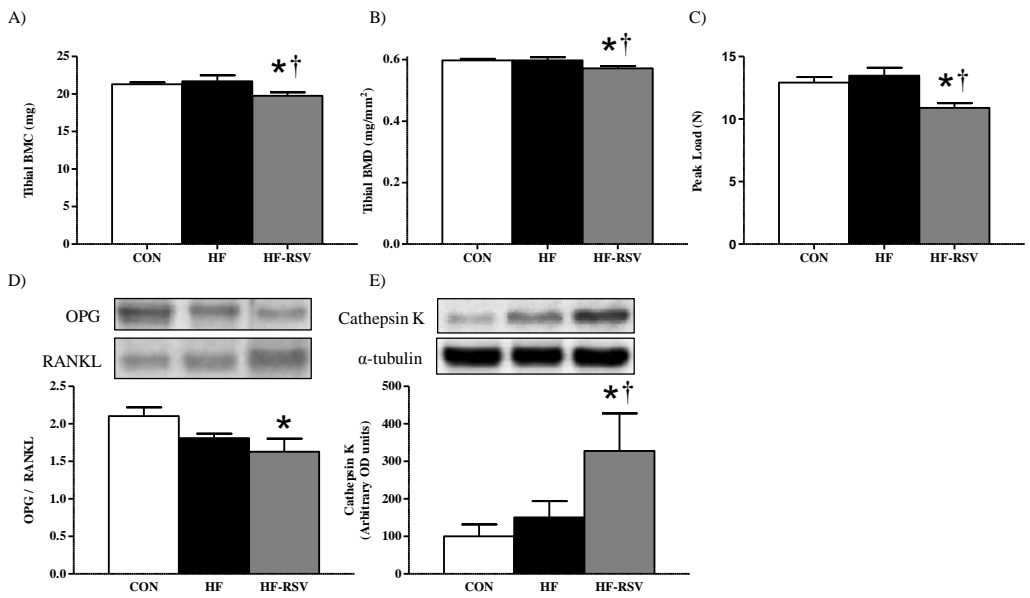

E)

E)
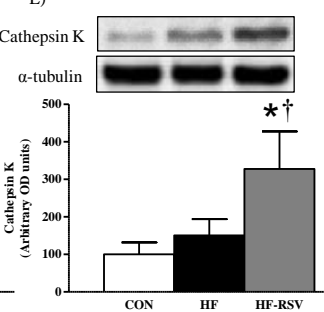

Figure 3

Figure 4. Markers of mitochondrial protein content in the tibia of mice fed a CON, HF, or HF-RSV diet. a) complex I, b) complex II, c) complex III, d) complex IV, e) complex V, f) representative blots for complexes I-V and $\alpha$-tubulin (loading control). Mice consuming a HF-RSV diet had higher protein content of complexes I, III, IV, and V than mice consuming a CON or HF diet. CON, control; HF, highfat; HF-RSV, high-fat-resveratrol. *, significantly different from CON. ${ }^{\dagger}$, significantly different from HF. Data expressed as Mean \pm SEM. $n=8-10 /$ group.

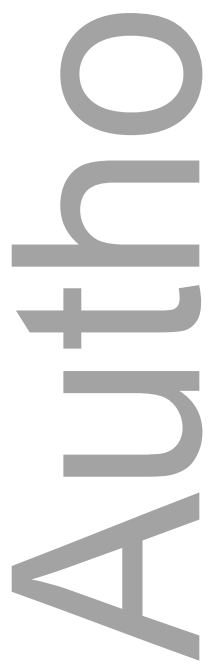

This article is protected by copyright. All rights reserved. 

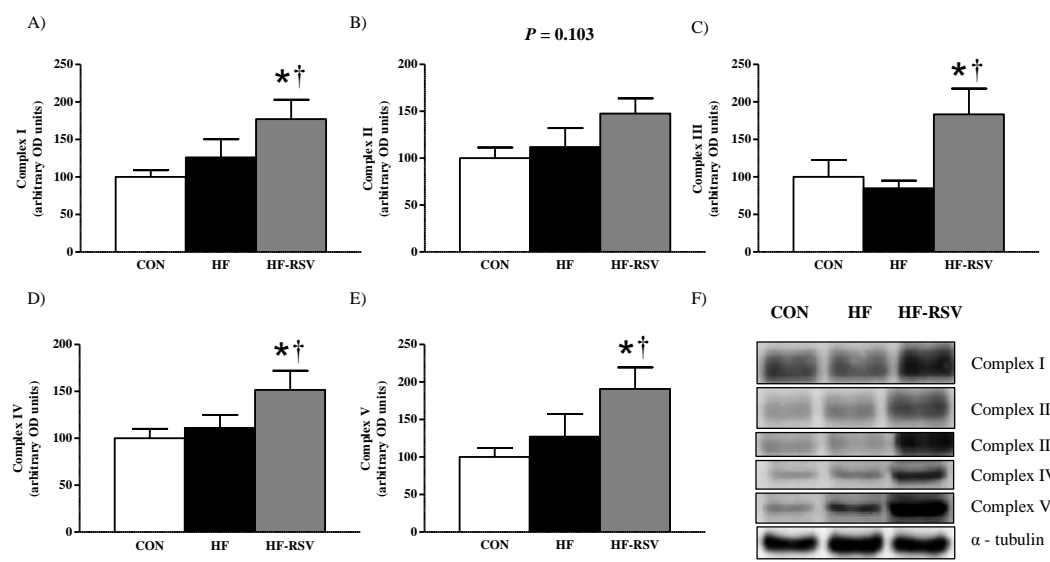

CON HF HF-RSV

Figure 4

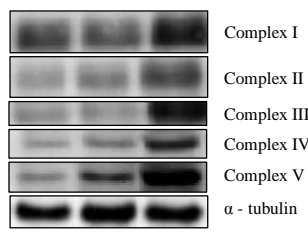

Figure 5. Markers of mitochondrial protein content in the tibia of RIP140 wild-type and KO mice.

a) complex I, b) complex II, c) complex III, d) complex IV, e) complex V, and f) representative blots of complexes I-V and $\alpha$-tubulin (loading control). RIP140 KO mice had higher complex I-V than WT controls. *, greater than WT. WT, wild-type; KO, knockout. Data expressed as mean \pm SEM. $\mathrm{n}=$ 5/group.
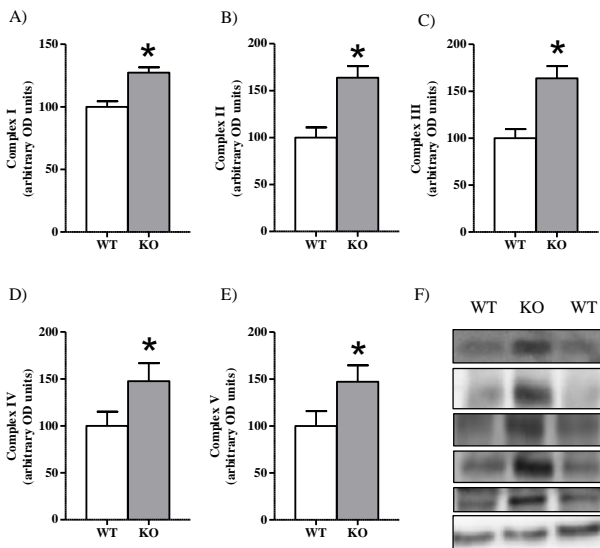

F) WT KO WT KO

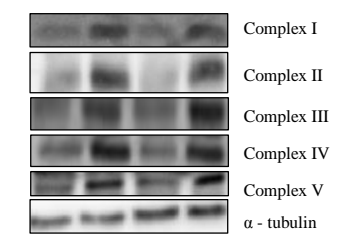

Figure 5

This article is protected by copyright. All rights reserved. 
Figure 6. Trabecular bone microarchitecture in the tibia of RIP140 wild-type and KO mice. a) Tb. BV/TV(\%), b) Tb. N, c) Tb.Th, d) Tb.Sp, and e) conn.D. RIP140 KO mice had lower Tb.BV/TV(\%), Tb.N, and Conn.D, coinciding with higher Tb.Sp without changes in trabecular thickness (Tb.Th). *, significantly different from WT. WT, wild-type; KO, knockout; Tb. BV/TV, bone volume/total volume; Tb. N, trabecular number; Tb.Th, trabecular thickness; Tb.Sp, trabecular separation; conn.D, connectivity density. Data expressed as mean \pm SEM. $n=5 /$ group.
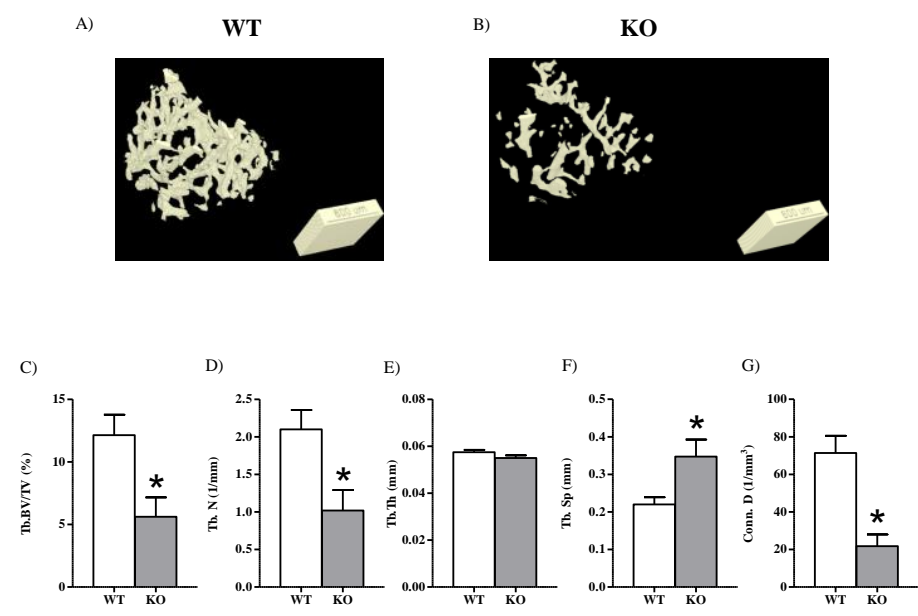

Figure 6

Figure 7. Bone mineral mass, bone strength, and markers of osteoclast activation and bone resorption in the tibia of RIP140 wild-type and KO mice. a) BMC, b) BMD, c) peak load, d) OPG/RANKL, e) Cathepsin K. RIP140 KO mice had lower BMD and peak load at the tibial midpoint than WT littermates. RIP140 KO mice had a lower OPG/RANKL ratio and higher cathepsin K than WT mice.*, significantly different from WT. WT, wild-type; KO, knockout; BMC, bone mineral content; BMD, bone mineral density. Data presented as mean \pm SEM. $n=5 /$ group.

This article is protected by copyright. All rights reserved. 

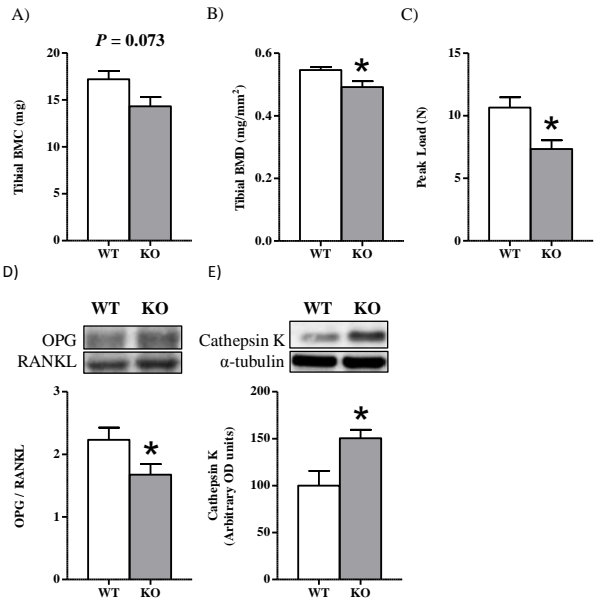

Figure 7

Figure 8. Correlations between bone mitochondrial content and BMC, BV/TV, peak load, and cathepsin $\mathrm{K}$.

There was a strong negative correlation between mitochondrial content and a) BMC, b) BV/TV (\%), and c) peak load, and positive correlation with d) cathepsin K. Data presented as mean \pm SEM.
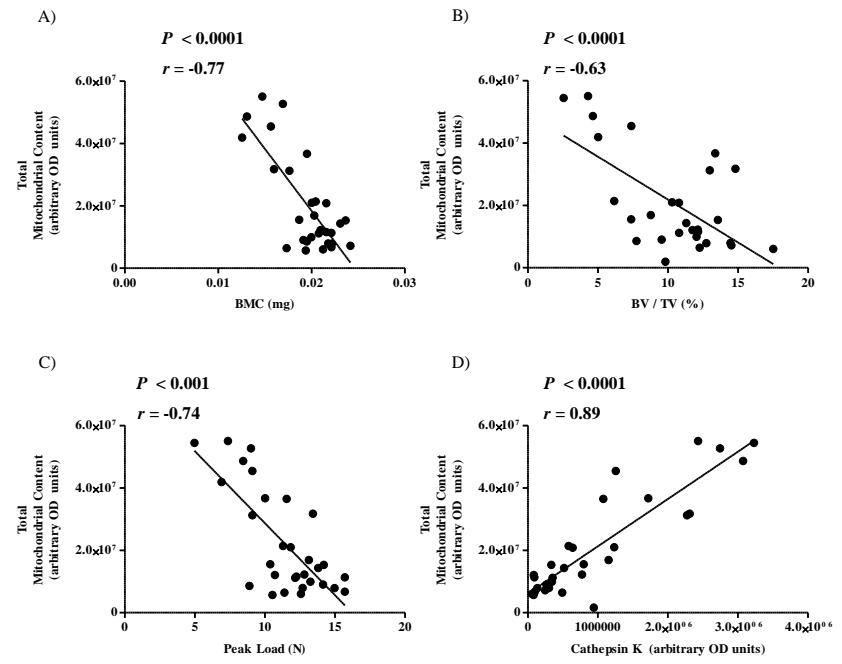

Figure 8

This article is protected by copyright. All rights reserved. 
Table 1. Basic characteristics and tibia outcomes from mice fed a CON, HF, or HF-RSV diet.

\begin{tabular}{|c|c|c|c|c|}
\hline \multirow[t]{2}{*}{ Outcome } & \multicolumn{3}{|c|}{ Diet } & \multirow[t]{2}{*}{$P$ Value } \\
\hline & CON & $\mathrm{HF}$ & HF-RSV & \\
\hline Body Weight (g) & $38.770 \pm 2.530$ & $46.860 \pm 1.510^{*}$ & $39.220 \pm 3.750 \dagger$ & $<0.001$ \\
\hline Food Intake (g/day & N/A & $2.44 \pm 0.150$ & $2.45 \pm 0.146$ & 0.991 \\
\hline \multicolumn{5}{|l|}{$\begin{array}{l}\text { Anthropometric } \\
\text { Measurements }\end{array}$} \\
\hline Length $(\mathrm{mm})$ & $17.904 \pm 0.112$ & $17.740 \pm 0.262$ & $17.620 \pm 0.134$ & 0.267 \\
\hline Width (mm) & $1.287 \pm 0.020$ & $1.290 \pm 0.010$ & $1.261 \pm 0.050$ & 0.332 \\
\hline Depth (mm) & $1.323 \pm 0.020$ & $1.248 \pm 0.020$ & $1.269 \pm 0.020$ & 0.134 \\
\hline Weight (g) & $0.054 \pm 0.001$ & $0.052 \pm 0.002$ & $0.050 \pm 0.001$ & 0.122 \\
\hline
\end{tabular}

Cortical Microarchitecture

$\begin{array}{ccccc}\text { Tt.Ar }\left(\mathrm{mm}^{2}\right) & 1.336 \pm 0.026 & 1.335 \pm 0.018 & 1.322 \pm 0.020 & 0.878 \\ \text { Ct.Ar }\left(\mathrm{mm}^{2}\right) & 0.783 \pm 0.013 & 0.772 \pm 0.015 & 0.776 \pm 0.013 & 0.844 \\ \text { Ct.Ar/Tt.Ar (\%) } & 58.686 \pm 0.612 & 57.822 \pm 0.598 & 58.688 \pm 0.259 & 0.378 \\ \text { Ct.Th (mm) } & 0.198 \pm 0.003 & 0.194 \pm 0.003 & 0.197 \pm 0.003 & 0.574 \\ & & & & \\ \text { Trabecular } & & & & \\ \text { Microarchitecture } & 1.558 \pm 0.022 & 1.574 \pm 0.045 & 1.527 \pm 0.033 & 0.610 \\ \text { TV }\left(\mathrm{mm}^{3}\right) & 0.196 \pm 0.013 & 0.186 \pm 0.016 & 0.155 \pm 0.012 & 0.085 \\ \text { BV }\left(\mathrm{mm}^{3}\right) & 2.378 \pm 0.053 & 2.436 \pm 0.047 & 2.494 \pm 0.046 & 0.244 \\ \text { SMI } & & & & \end{array}$

*, significantly lower than CON. †, significantly lower than HF. Tt.Ar, total cross-sectional area inside the

This article is protected by copyright. All rights reserved. 
periosteal envelope; Ct.Ar, cortical bone area; Ct.Ar/Tt.Ar, cortical area fraction; Ct.Th, cortical thickness; TV, total volume; BV, bone volume; SMI, structure model index. CON, control; HF, high-fat; HF-RSV, high-fatresveratrol; N/A, not available. Data is expressed as mean \pm SEM. $n=8-10 /$ group.

Table 2. Tibia outcomes from RIP140 WT and KO mice.

\begin{tabular}{cccc}
\hline Outcome & \multicolumn{2}{c}{ Genotype } & P Value \\
\hline & RIP140 WT & RIP140 KO & \\
\hline Anthropometric Measurements & & & \\
Length (mm) & $17.600 \pm 0.542$ & $17.132 \pm 0.310$ & 0.276 \\
Width (mm) & $1.070 \pm 0.058$ & $1.016 \pm 0.076$ & 0.360 \\
Depth (mm) & $1.122 \pm 0.091$ & $1.104 \pm 0.073$ & 0.739 \\
Weight (g) & $0.047 \pm 0.05$ & $0.041 \pm 0.006$ & 0.164 \\
Cortical Microarchitecture & & & \\
Tt.Ar $\left(\mathrm{mm}^{2}\right)$ & $0.928 \pm 0.046$ & $0.828 \pm 0.078$ & 0.168 \\
Ct.Ar $\left(\mathrm{mm}^{2}\right)$ & $0.613 \pm 0.033$ & $0.529 \pm 0.053$ & 0.249 \\
Ct.Ar/Tt.Ar (\%) & $62.424 \pm 0.985$ & $63.735 \pm 0.458$ & 0.294 \\
Ct.Th (mm) & $0.193 \pm 0.006$ & $0.186 \pm 0.009$ & 0.547
\end{tabular}


Trabecular Microarchitecture

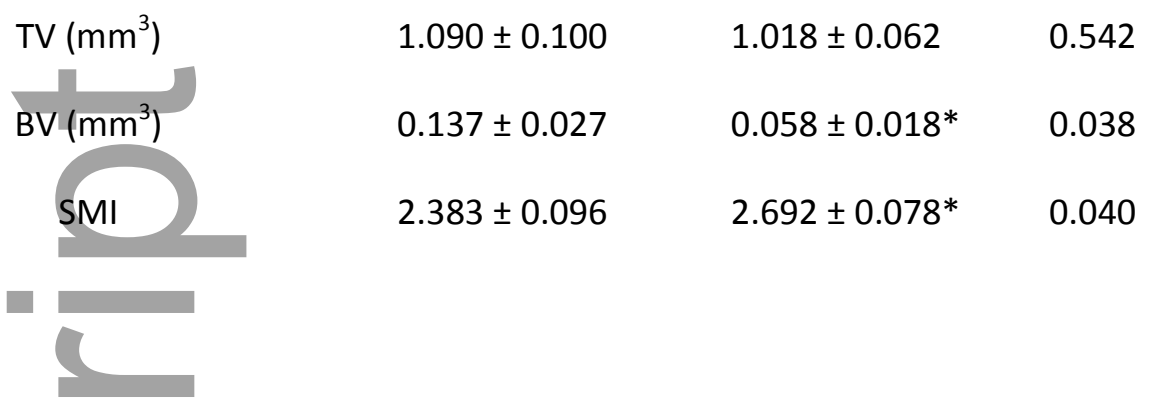

*, significantly different from WT. Tt.Ar, total cross-sectional area inside the periosteal envelope; Ct.Ar, cortical bone area; Ct.Ar/Tt.Ar, cortical area fraction; Ct.Th, cortical thickness; TV, total volume; BV, bone volume; SMI, structure model index. WT, wild-type; $K O$, knockout. Data is expressed as mean \pm SEM. $n=3-5$ /group.

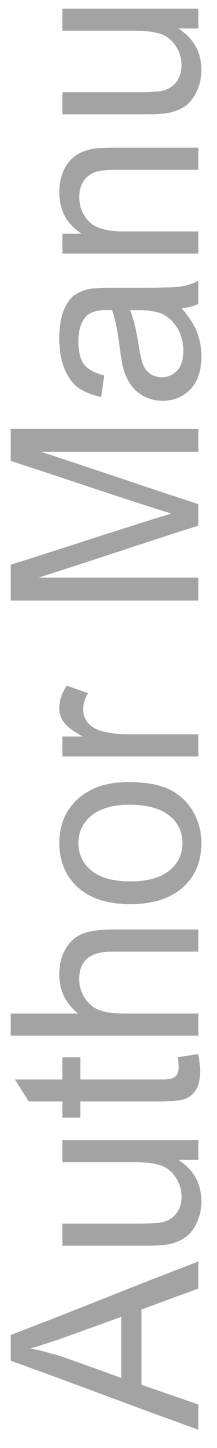

This article is protected by copyright. All rights reserved. 


\section{University Library}

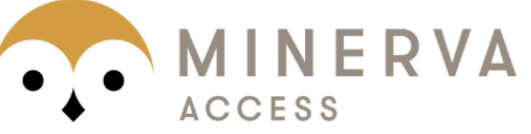

A gateway to Melbourne's research publications

Minerva Access is the Institutional Repository of The University of Melbourne

Author/s:

Miotto, PM;Frendo-Cumbo, S;Sacco, SM;Wright, DC;Ward, WE;Holloway, GP

Title:

Combined high-fat-resveratrol diet and RIP140 knockout mice reveal a novel relationship between elevated bone mitochondrial content and compromised bone microarchitecture, bone mineral mass, and bone strength in the tibia.

Date:

2016-09

Citation:

Miotto, P. M., Frendo-Cumbo, S., Sacco, S. M., Wright, D. C., Ward, W. E. \& Holloway, G. P. (2016). Combined high-fat-resveratrol diet and RIP140 knockout mice reveal a novel relationship between elevated bone mitochondrial content and compromised bone microarchitecture, bone mineral mass, and bone strength in the tibia.. Mol Nutr Food Res, 60 (9), pp.1994-2007. https://doi.org/10.1002/mnfr.201500870.

Persistent Link:

http://hdl.handle.net/11343/291197 